\title{
Sex Effects of Marijuana on Brain Structure and Function
}

\author{
Ariel Ketcherside $^{1} \cdot$ Jessica Baine $^{1} \cdot$ Francesca Filbey $^{1}$ \\ Published online: 7 July 2016 \\ (C) The Author(s) 2016. This article is published with open access at Springerlink.com
}

\begin{abstract}
Background Tetrahydrocannabinol $\left(\Delta^{9}-\mathrm{THC}\right)$, the primary ingredient in marijuana, exerts its effects across several neurological and biological systems that interact with the endocrine system. Thus, differential effects of $\Delta^{9}$-THC are likely to exist based on sex and hormone levels.

Methods We reviewed the existing literature to determine sexbased effects of $\Delta^{9}$-THC on neural structure and functioning. Results The literature demonstrates differences in male and female marijuana users on brain structure, reward processing, attention, motor coordination, and sensitivity to withdrawal. However, inconsistencies exist in the literature regarding how marijuana affects men and women differentially, and more work is needed to understand these mechanisms. While extant literature remains inconclusive, differentiation between male and female marijuana users is likely due to neurological sexual dimorphism and differential social factors at play during development and adulthood.

Conclusions Sex has important implications for marijuana use and the development of cannabis use disorders and should be considered in the development of prevention and treatment strategies.
\end{abstract}

Keywords Cannabis · Neuroimaging · MRI · Gender · Gendered treatment $\cdot$ THC $\cdot$ Sexual dimorphism

This article is part of the Topical Collection on Cannabis

Francesca Filbey

Francesca.Filbey@utdallas.edu

1 Center for BrainHealth, School of Behavioral and Brain Sciences, University of Texas at Dallas, 2200 W. Mockingbird Lane, Dallas, TX 75235, USA

\section{Introduction}

Marijuana continues to be the most widely used illicit substance in the world $[1,2]$. Similar to other substances of abuse [3], there are more male marijuana users in the USA (e.g., $54.1 \%$, [4]) than females historically; however, recent trends suggest that the number of female users is increasing, while the number of male users is remaining stable [5]. Interestingly, female users have been reported to develop cannabis use disorders (CUDs) more quickly after initiation of marijuana use than males (i.e., "telescoping") [6-8], suggesting potential sex differences underlying the effects of $\Delta^{9}$-THC. These sex differences in the effects of $\Delta^{9}$-THC can be attributed to the interaction of the endocannabinoid and endocrine systems, as the endocannabinoid system is widely known for its modulatory role in endocrine functioning [9]. Alternatively, premorbid cognitive differences between males and females may also contribute to the sex differences observed following marijuana use. Nevertheless, these sex differences implicate the need for different prevention and treatment strategies for males and females (Table 1).

\section{Interaction Between Marijuana and Hormones: Mechanism for Sex Effects}

The endocannabinoid system modulates the hypothalamic pituitary gonadal (HPG) axis. Animal studies have demonstrated that when $\Delta^{9}$-THC binds to cannabinoid receptors on gonadotropin-releasing hormone $(\mathrm{GnRH})$ releasing cells in the hypothalamus, it has differential downstream effects in men and women. In females, there have been reports of $\Delta^{9}$ THC both stimulating and suppressing the secretion of luteinizing hormone, which not only indicates variable effects at different stages of the menstrual cycle [39-41]. 
Table 1 Neurocognitive differences across male and female marijuana users

\begin{tabular}{|c|c|c|}
\hline Authors & Modality & Participants \\
\hline Anderson et al. (2010) [10] & $\begin{array}{l}\text { Double-blind, placebo- } \\
\text { controlled (active or placebo } \\
\text { marijuana cigarette) }\end{array}$ & 50 males, 35 females \\
\hline Anderson et al. (2010) [11] & $\begin{array}{l}\text { Attention, cognitive flexibility, } \\
\text { time estimation, and } \\
\text { visuospatial processing } \\
\text { tasks }\end{array}$ & $\begin{array}{l}70 \text { occasional marijuana users } \\
(50 \% \text { male })\end{array}$ \\
\hline Block et al. (1991) [12••] & $\begin{array}{l}\text { Blood sample collection, } \\
\text { hormone analyses, and } \\
\text { substance use questionnaires }\end{array}$ & $\begin{array}{l}93 \text { males and } 56 \text { females with } \\
\text { either frequent, moderate, } \\
\text { infrequent, or no marijuana } \\
\text { use }\end{array}$ \\
\hline Block et al. (2000) [13] & Imaging: volume & $\begin{array}{l}18 \text { adult marijuana users ( } 50 \% \\
\text { male) vs. } 13 \text { controls ( } 46 \% \\
\text { male) }\end{array}$ \\
\hline Buckner et al. (2012) [14] & $\begin{array}{l}\text { Multi-site, marijuana smoking } \\
\text { history, MMM, MPS, SIAS }\end{array}$ & $\begin{array}{l}174 \text { current marijuana users } \\
\quad(57.5 \% \text { male })\end{array}$ \\
\hline
\end{tabular}

Putative difference between males and females

No sex differences were observed.

trolled (active or placebo

ood sample collection, hormone analyses, and

maging: volume

history, MMM, MPS, SIAS

SCID DSM-IV-TR (SCID I/

$\mathrm{NP}$ ) and self-report ques-

tionnaires

Cooper and Haney (2014) [16]

Copersino et al. (2010) [17]

Cousijn et al. (2012) [18]

Crane et al. (2013) [19••]

Data combined from four double-blind, within-subject studies using active vs. inactive marijuana

Retrospective self-report measures

Imaging: voxel-based morphometry (VBM)

Neuropsychological tests

Self-report and BART task over period of grades $8-12$

Gillespie et al. (2011) [21]

Guxens et al. (2007) [22]

Hernandez-Avila et al. (2004) [7]
Structured interviews on DSMIV criteria of CUD

Cohort study, self-administered lifestyle questionnaire
123 undergraduates $(40.7 \%$ male)

35 male vs. 35 female marijuana users

104 non-treatment seeking adult marijuana smokers ( $78 \%$ male)

33 heavy marijuana users (64\% male) vs. 42 controls (62\% male)

44 male vs. 25 female marijuana users

115 male and 89 female adolescents

7316 adult male and female twins

1056 adolescents $(52.2 \%$ male)

271 substance-dependent patients ( $42 \%$ male), 38 of those were marijuanadependent ( $47 \%$ male)
No sex differences were observed, though the authors discuss a higher rate of study attrition in females.

No effect of chronic marijuana use on sex hormones (FSH, prolactin, LH, or testosterone) was found.

Sex differences were detected in several brain volume measures.

Social anxiety in men was positively related to CUD problems and conforming and coping motives. Females demonstrated a positive correlation between social anxiety with social motives only.

Symptoms of social anxiety disorder were correlated with CUD symptoms in women only, and peer use of both alcohol and marijuana was found to moderate this relationship.

Women reported higher ratings of abuse-related effects relative to men under the active marijuana condition, but men and women did not differ in self-reported ratings of intoxication.

Females were more likely than males to report a withdrawal symptom (upset stomach, increased sex drive, marijuana craving).

No interactions of either gray matter or white matter differences and sex was found between either of the groups.

Earlier age of initiated use was related to less education, lower IQ, fewer years of maternal education, and poorer episodic memory in women only, but more lifetime marijuana use in men.

An interaction of sex and disinhibition suggested that only males who selfreported greater disinhibition showed greater increases in their marijuana use.

Lower factor loadings for women suggest that legal problems may discriminate better among men.

Fewer factors predicting marijuana use were found in males than in females. Predictive variables reflecting type of school, family situation, and academic performance were present only among girls.

No sex effects on age of onset for marijuana users were found. Women who were marijuana-dependent reported less pretreatment years of regular marijuana consumption, 
Table 1 (continued)

\begin{tabular}{lll}
\hline Authors & Modality & $\begin{array}{l}\text { Participants } \\
\text { Putative difference between males } \\
\text { and females }\end{array}$
\end{tabular}

Johnson et al. (2015) [23]

Jones et al. (2008) [24]

Khan et al. (2013) [6]

Lisdahl and Price (2012) [25]

McDonald et al. (2003) [26]

McQueeny et al. (2011) [27]

Medina et al. (2009) [28]

Noack et al. (2011) [29]

Pedersen et al. (2001) [30] characteristics
Neuropsychological tests

Data from National Youth Risk Behavior Survey (YRBS) 1999-2013 assessment of blood

Data from the National Epidemiologic Survey of Alcohol and Related Conditions 2001-2002

Double-blind, placebo conditions, 7.5 or $15 \mathrm{mg}$ THC capsule. Stop, Go/NoGo, delay discounting, and time estimation tasks Imaging: sMRI
Enzyme immunoassay

\author{
115,379 adolescents (50\% \\ male) \\ 8794 adults (94\% male)
}

3297 US adults diagnosed with lifetime CUD (63\% male)

23 marijuana users ( $44 \%$ male) vs. 35 controls ( $50 \%$ male)

37 healthy recreational marijuana users ( $49 \%$ male)

35 marijuana users vs. 47 controls (both groups $77 \%$ male)

Imaging: MRI

Internet survey of use compared to men. Women were less likely than men to be diagnosed with current marijuana dependence.

Sex differences were observed to substantially decrease over time for each race/ethnicity group.

For DUID ${ }^{\text {a }}$ suspects, the number of men far exceeded that of women, and women were older than the men. Blood THC concentration was higher in men than in women.

Women with CUD presented more mood and anxiety disorders and had an increased risk for externalizing disorders. Men with CUD had an increased risk of being diagnosed with an SUD, antisocial personality disorder, or a psychiatric disorder. Men with CUD were older at remission, used more joints, and reported more CUD symptoms than women.

Female users were found to have an earlier age of onset for regular marijuana use. Male users also demonstrated a stronger relationship between both poor sequencing ability, psychomotor speed, and increased marijuana use, even though both male and female users had similar levels of past year marijuana use.

No significant sex differences in performance on the impulsivity measures were observed.

Female marijuana users had larger right amygdala volumes and more internalizing symptoms than female controls, while male users had similar volumes to male controls. For female controls and males, worse mood/anxiety was linked to smaller right amygdala volume, whereas more internalizing problems were associated with greater right amygdala volume in female marijuana users only.

Female users presented more lifetime drinking episodes and symptoms of alcohol dependence. Male users showed smaller PFC volumes while female users displayed larger PFC volumes compared to their same-sex controls.

Marijuana use with a water pipe was more often reported by males, while use before sleep was more often reported by females. When rating the social contexts of their marijuana use, women reported more use "with strangers" than men. 
Table 1 (continued)

Authors

Modality

Participants

Longitudinal study of conduct

and cannabis use

Pope et al. (1997) [31]

Price et al. (2015) [32]

Roser et al. (2009) [33]

Schepis et al. (2011) [34]

Skosnik et al. (2006) [35]

Tu et al. (2008) [36]
Visuospatial memory task

Imaging: MRI

Double-blind, placebocontrolled cross-over study: psychomotor performance using finger-tapping test series

Cross-sectional statewide survey of adolescent risk behavior

EEG: visual function via SSVEP conducted in 2004
Cross-sectional survey
25 heavy marijuana users vs. 30 light marijuana users

27 marijuana users (56\% male) vs. 32 controls ( $44 \%$ male)

24 healthy volunteers (50\% male)

4523 public high school students (48.2\% male)

17 marijuana users (59\% male) vs. 16 healthy drug naïve controls (38\% male)

8225 students grade $7-12$

(50\% male)
Putative difference between males and females

Conduct problems had an impact on marijuana initiation, with a noticeably stronger effect in females. For females, covert and aggressive conduct problems had robust effects, while in males, serious conduct had a moderate effect.

Heavy marijuana-using women have impaired memory compared to light marijuana-using women, but there was no effect in men.

No significant sex interactions were found.

Males showed faster left-handed taps than females after $\Delta^{9}$-THC condition. Females showed greater variability in tapping speed after THC administration compared to placebo. No sex differences in the tapping frequencies under the placebo condition were found. Female subjects revealed a higher AIR-Scale score under $\Delta^{9}$-THC, but not under MJ extract. Overall, females performed worse than males for the left-hand tapping frequencies, demonstrated higher levels of plasma THC metabolites, and reported greater perception of intoxication compared to males.

African-American males and Caucasian females were more likely to use marijuana than their counterparts of the same race, while Asian females were less likely to use. Males with depression and anhedonia in the past year had greater odds of marijuana use in the past year as well. Overall, females also demonstrated more rapid transition from initiation to regular marijuana use.

No sex differences were observed in the marijuana group for any substance use data. A main effect of sex was observed, indicating that females displayed a larger SSVEP response. A sex-by-group interaction was observed at $18 \mathrm{~Hz}$, indicating that marijuana use reduced $18 \mathrm{~Hz}$ spectral power in females, but not in males. Overall, these findings suggest that attention may be more impaired in male marijuana users.

Aboriginal boys but not girls were more likely to use marijuana. Marijuana use was associated with higher school grade among boys, but not girls, and girls who used marijuana were more likely to report poorer mental health than boys. 
Table 1 (continued)

\begin{tabular}{|c|c|c|c|}
\hline Authors & Modality & Participants & $\begin{array}{l}\text { Putative difference between males } \\
\text { and females }\end{array}$ \\
\hline Wetherill et al. (2015) [37••] & Imaging: fMRI & $\begin{array}{l}44 \text { treatment-seeking, } \\
\text { marijuana-dependent adults } \\
(61 \% \text { male })\end{array}$ & $\begin{array}{l}\text { Both sexes responded similarly to } \\
\text { backward-masked marijuana cues }> \\
\text { neutral cues, but for women, activity } \\
\text { in the insula during this task corre- } \\
\text { lated with MJ craving, while left } \\
\text { OFC was inversely correlated with } \\
\text { craving. For men, activity in the } \\
\text { striatum during this task correlated } \\
\text { with craving. }\end{array}$ \\
\hline Zalesky et al. (2012) [38] & $\begin{array}{l}\text { imaging: MRI axonal fiber } \\
\text { connectivity }\end{array}$ & $\begin{array}{l}59 \text { marijuana users ( } 47 \% \text { male) } \\
\text { vs. } 33 \text { matched controls } \\
\text { ( } 42 \% \text { male) }\end{array}$ & $\begin{array}{l}\text { No significant sex differences or sex by } \\
\text { group interactions were found. }\end{array}$ \\
\hline
\end{tabular}

${ }^{\text {a }}$ Driving under the influence of drugs

Studies in humans have been inconsistent, however. For example, in chronic male marijuana users, decreased testosterone has been shown [42], but has not been replicated [39]. Further, one study found no effect of chronic marijuana use on sex hormones in males and females, including follicle stimulating hormone, prolactin, luteinizing hormone, or testosterone $[12 \bullet \bullet]$. In all, hormonal differences between men and women should be considered as a mechanism for differential effects of $\Delta^{9}$-THC on brain structure and function.

\section{Sex Differences on Brain Structures of Marijuana Users}

Sexual dimorphism in the human brain is widely reported. For example, brain development is different between sexes such that total brain size peaks between 10 and 11 years of age in females, while total brain size peaks at 14 to 15 years of age in males [19••, 43]. Regarding overall brain tissue, an interaction between white and gray matter development and sex has been noted where prefrontal cortex (PFC) gray matter volume in females peaks 1-2 years earlier than in males, while males demonstrate greater age-related increases in white matter [44]. Brain regions specific to reward also develop differently between males and females. Amygdala volume increases in males as a function of androgen receptor density, while estrogen receptor density in the female hippocampus results in greater female hippocampal growth [45]. Thus, sex differences in brain structure may create a variable environment for $\Delta^{9}$-THC especially during periods of neural development when the brain is more vulnerable to structural and/or functional changes $[4,19 \bullet \cdot]$, such as in synaptic pruning. For example, in a study of adolescent (age 16-18) marijuana users, females had greater PFC volume than males, which was associated with impaired executive functioning, suggesting that sex moderates the relationship between marijuana use and
PFC volume [28]. Similarly, another study found larger amygdala volumes in female adolescent marijuana users relative to non-users, which was not observed in male users [27]. Other studies, however, have not found similar interactions with sex [32].

In adults, while marijuana use has been associated with alterations in specific brain areas [46], differences between sexes have only been found on the whole brain level (e.g., total whole brain volume). In a study by Block and colleagues (2000), no effect of user vs. non-user status or sex on total brain tissue volume was found, although total intracranial volume, total intracranial tissue, cerebrospinal fluid (CSF), and combined cerebral gray matter were found to be higher in male marijuana users than in female marijuana users [13]. In a study using voxel-based morphometry (VBM), Cousijn et al. (2012) found no interactions of either gray matter or white matter differences and sex between groups [18].

In sum, the literature on sex-based differences in brain structure is limited. However, the existing studies suggest that male and female marijuana users have differences in brain structure, particularly in regions involved in reward processing. Differences in adolescent male and female users may be due to the sensitivity of this neural developmental period to $\Delta^{9}$-THC as alterations have been noted in mesocorticolimbic regions. However, there are inconsistencies in the literature. Future studies in adult marijuana users are needed to determine whether differences exist in localized areas of the brain.

\section{Sex Differences on Brain Function of Marijuana Users}

The ubiquitous nature of the endocannabinoid system suggests that effects of $\Delta^{9}$-THC may span wide-ranging neural processes. In this article, we focus on each of the cognitive 
processes most widely examined in marijuana users and report sex-based differences demonstrated in the literature.

\section{Craving}

Cannabinoids act directly on the nucleus accumbens triggering the release of dopamine in the mesolimbic "reward" circuit in the same manner as other drugs of abuse [47]. Though the mechanism is the same in males and females, evidence suggests differences in activation. For example, Cooper et al. (2014) demonstrated that despite no difference in levels of intoxication, females reported greater subjective positive effects than males (i.e., feeling "good," and that they would "take it again") [16]. Similarly, while both sexes experience subjective craving, Wetherill et al. (2015) demonstrated that this process might occur differently between treatmentseeking marijuana-dependent males and females [37••]. In their study, they used a backward masking of marijuana cues that allows for subconscious but not conscious processing of visual stimuli. Preliminary analyses showed greater response in the striatum, left hippocampus and amygdala, and left lateral orbitofrontal cortex (OFC) of females compared to males when exposed to the masked marijuana cues relative to masked neutral cues. Thus, females appear to exhibit greater cortical involvement in valuation of cues relative to males. Further analyses also revealed differences in correlations between neural response to marijuana cues and subjective craving with the most notable difference a negative correlation in the left lateral OFC in female users and an absence of a negative correlation in males. The negative correlation in females was interpreted as top-down cognitive control during exposure to cues, or the incorporation of previously learned patterns via higher-order (i.e., incorporating more information globally) brain regions in processing these cues. The lack of such processing in males suggests the lack of recruitment of these higher-order brain regions. In conclusion, the authors suggest that incorporation of top-down neural functioning may be a viable treatment approach, as pattern recognition may help those with CUDs (presumably males more than females) identify the deleterious effects of marijuana use $[37 \bullet \bullet]$.

\section{Inhibitory Control}

Impulsivity is a known risk fact for CUD [48] and may differ between the sexes in some components (i.e., sensation seeking) but not others (i.e., delay discounting) [49]. The concept of impulsivity is broad and encompasses several cognitive domains that characterize one's ability to control one's behavior. In a study of adolescent marijuana users, an interaction between self-reported behavioral disinhibition (a composite score of measures of impulsivity and sensation seeking) and sex was found, such that males with higher self-reported disinhibition were more likely to use marijuana than females (with higher self-reported disinhibition?) [20]. However, because impulsivity wanes through maturation, it would be important to determine if this trend continues in adulthood [50]. Currently, existing studies in adult marijuana users do not demonstrate sex differences in domains of impulsivity during acute $\Delta^{9}$-THC intoxication. For example, McDonald and colleagues did not find differences between adult male and female marijuana users on inhibitory control tasks including (i) the Stop Signal task, which measures the motor response inhibition to an ongoing motor response, (ii) the Go/No-Go task, which measures the ability to withhold a motor response to prepotent stimuli, or (iii) the Delay Discounting Task, which measures the cognitive ability to delay immediate, smaller rewards for larger, later rewards [26]. These inhibitory control functions may also be referred to as "stopping impulsivity" and "waiting impulsivity" respectively, and while both circuits implicate mesolimbic dopaminergic circuitry, the former involves motor regions while the latter involves top-down, cortical control of behavior [51]. The lack of sex effects in these domains suggests that $\Delta^{9}$-THC intoxication does not have unique sex effects on either motor or cognitive control [51].

In sum, the broad concept of impulsivity need to be disentangled in order to better understand the sex effects of marijuana on domains of impulsivity. However, in adolescents, general impulsive behavior is differentially linked to marijuana use between sexes.

\section{Motor Coordination}

Given the abundance of cannabinoid receptors in brain motor control regions such as the basal ganglia and cerebellum, it is not surprising that studies have shown acute effects of THC on motor coordination and control, including how these may differ between the sexes. One task used to examine this is a finger-tapping frequency task that is designed to examine fine motor coordination. Using this task, Roser and colleagues (2009) found that males demonstrate significantly faster left-hand tapping than females after $\Delta^{9}$-THC administration, but not right (dominant) hand tapping [33]. Females in this study also reported higher subjective intoxication ratings and had higher concentrations of THC metabolites in their blood after being administered the same amount of $\Delta^{9}$-THC as males. The authors concluded that, while no effect was seen with the dominant hand, perhaps greater intoxication in females is related to greater "functional instability" in their nondominant hand. Another study examining neuropsychological functioning in marijuana using adults found that male users demonstrated a stronger relationship between both poor sequencing ability, psychomotor speed, and increased marijuana use, even though both male and female users had similar levels of past year marijuana use [25]. 
Aspects of motor coordination can also be explored using driving tasks, although other cognitive processes such as attention likely confound overall performance. For example, Anderson et al. (2010) tested acute effects of $\Delta^{9}$-THC before and after smoking a marijuana or placebo cigarette [10] during a distracted driving simulator task. Participants who received the placebo cigarettes demonstrated learning effects wherein they exhibited improved performance the second time the test was administered, whereas participants administered marijuana cigarettes did not. However, no sex differences were noted in performance. The lack of sex differences in the driving task suggests that complex motor skills may not be as sensitive to sex effects of $\Delta^{9}-$ THC as in those that measure fine motor coordination [10].

In sum, studies of motor coordination suggest that fine motor coordination differences between sexes may be related to degree of intoxication. Future studies should control for levels of intoxication and determine whether effects remain despite similar intoxication levels. More complex motor tasks should control for confounding effects of higher-order processes that may influence fine motor coordination.

\section{Memory}

The literature widely supports the effects of marijuana on memory, particularly short-term memory in intoxicated individuals [52]. Studies focused on sex differences on memory functions in marijuana users suggest domain-specific effects. However, such findings have not been consistent. For instance, Pope et al. (1997) showed that heavy (smoked 29 out of the past 30 days) marijuana using females had impaired memory of visual checkerboard patterns compared to light (smoked one out of the past 30 days) marijuana using females, whereas no difference between heavy- and light-using males were found following a supervised abstinence period of at least $19 \mathrm{~h}$. In addition, no difference was found between the sexes (i.e., heavy-using women vs. heavy-using men, or lightusing women vs. light-using men) [31]. On the contrary, in a study examining acute effects of marijuana, Anderson et al. (2010) did not find a difference in visuospatial processing between sexes [11]. Similar to motor coordination, inconsistencies in differences in memory performance could be due to differences in levels of intoxication.

\section{Attention}

While impaired attention has been documented in marijuana users [53], only one study to date documents differences between the sexes. Skosnik and collegues (2006) used electroencephalography (EEG) to examine attention via the steadystate visual evoked potential (SSVEP), particularly the N160 response, in current (at least one use per week) marijuana users. This response to visual stimuli is thought to measure attention by means of visual processing. Overall, the N160 response was lower in marijuana users compared to controls. Additionally, it was found to be lowest in the male marijuana users [35]. This suggests that attention may be more impaired in male marijuana users than in female users. However, two caveats were noted. First, males reported using 11.2 joints per week on average, which is greater than females, who averaged 9.3. Second, females' menstrual cycle was not documented, which may affect visual processing [54].

There is limited literature suggesting general differences in attention between males and females, particularly on memory performance during divided attention [55], and during attention related to visual motor processing [56]. In light of this, given marijuana's known general effects on attention, future work should examine potential differences between men and women both during acute intoxication and after chronic marijuana use.

\section{Conclusions and Future Directions}

Although the number of studies that directly examine sex differences in marijuana users is limited (fewer than 30), the existing literature shows differences in brain structure and function. Differences in brain structure and function between male and female marijuana users show a complex picture that likely reflects the complicated sexual dimorphism that occurs in all biological systems. Thus, differential effects of marijuana based on sex may be different on multiple levels.

Our review revealed inconsistencies in the existing literature, which could be attributed to a number of factors. First, there may be underlying risk factors that are independent of sex. For example, risk factors for CUDs may be more prevalent within the subpopulation of women who do use marijuana and thus contribute toward CUDs. This is supported by the greater hedonic responses to marijuana reported by female users. However, the neural processes involved in this etiology are complex and require extensive further examination. Compounding the unique effects of marijuana on sex are multiple factors that have yet to be delineated. Studies have already begun to illustrate the importance of age of initiation of use [57]. Male and female brains develop at different rates and in different ways invariably resulting in differential effects of marijuana on each sex depending on age of exposure. Another limitation in studies of acute effects of THC in sexes is due to differences in subjective measures of intoxication. For example, D'Souza and colleagues identified variable reports in euphoria, perceptual alterations, feelings of anxiety, and disorganization of thoughts among acutely intoxicated participants. Variability between participants in these symptoms would likely confound effects of THC on outcome measures [58].

A final consideration worth noting is the self-selection in participants that are likely to confound study findings. As 
marijuana is still illegal in the majority of the USA, recruitment for marijuana using participants has its challenges. There may be differential effects of social norms on men and women that result in imbalanced representations between these sexes. One study revealed that "attempts were made to recruit equal numbers of men and women, however, fewer women expressed interest in participating in the study" [28]. Additionally, the women that do overcome aversion to admitting they use marijuana may demonstrate different personality traits than female marijuana users at large and could therefore be skewing the documented data. Thus, there may be limited generalizability in existing findings.

In conclusion, the differential effects of marijuana on the structure and function of male and female brains remains elusive. Some effects have been shown concerning attention, motor coordination, and impulsivity, but further work is needed to disentangle the mélange of variables affecting sex differences in marijuana users. Future directions should include controls for quantity of marijuana and ideally THC/CBD concentration, as well as females' menstrual cycles, and age of initiation. Clinicians should consider the differences put forth by existing literature.

\section{Compliance with Ethical Standards}

Conflict of Interest Ariel Ketcherside, Jessica Baine, and Francesca Filbey declare that they have no conflict of interest.

Human and Animal Rights and Informed Consent This article does not contain any studies with human or animal subjects performed by any of the authors.

Open Access This article is distributed under the terms of the Creative Commons Attribution 4.0 International License (http:// creativecommons.org/licenses/by/4.0/), which permits unrestricted use, distribution, and reproduction in any medium, provided you give appropriate credit to the original author(s) and the source, provide a link to the Creative Commons license, and indicate if changes were made.

\section{References}

Papers of particular interest, published recently, have been highlighted as:

•• Of major importance

1. United Nations Office on Drug and Crime (2011) World drug report.

2. Choo EK et al. The impact of state medical marijuana legislation on adolescent marijuana use. J Adolesc Health Off Publ Soc Adolesc Med. 2014;55:160-6.

3. Degenhardt L, Hall W. Extent of illicit drug use and dependence, and their contribution to the global burden of disease. Lancet. 2012;379:55-70.

4. SAMHSA (2014) Results from the 2013 national survey on drug use and health: summary of national findings. at $<\mathrm{http}$ ://www.samhsa.
gov/data/sites/default/files/NSDUHresultsPDFWHTML2013 /Web/NSDUHresults2013.pdf $>$

5. SAMHSA (2008) Results from the 2007 national survey on drug use and health: national findings. at $<\mathrm{http}: / /$ archive.samhsa. gov/data/NSDUH/2k7nsduh/2k7Results.htm>

6. Khan SS et al. Gender differences in cannabis use disorders: results from the national epidemiologic survey of alcohol and related conditions. Drug Alcohol Depend. 2013;130:101-8.

7. Hernandez-Avila CA et al. Opioid-, cannabis- and alcoholdependent women show more rapid progression to substance abuse treatment. Drug Alcohol Depend. 2004;74:265-72.

8. Van Etten ML, Anthony JC. Comparative epidemiology of initial drug opportunities and transitions to first use: marijuana, cocaine, hallucinogens and heroin. Drug Alcohol Depend. 1999;54:117-25.

9. Pagotto $U$ et al. The emerging role of the endocannabinoid system in endocrine regulation and energy balance. Endocr Rev. 2006;27: 73-100.

10. Anderson BM et al. Sex differences in the effects of marijuana on simulated driving performance. J Psychoactive Drugs. 2010;42:19-30.

11. Anderson BM et al. Sex, drugs, and cognition: effects of marijuana. J Psychoactive Drugs. 2010;42:413-24.

12.• Block RI et al. Effects of chronic marijuana use on testosterone, luteinizing hormone, follicle stimulating hormone, prolactin and cortisol in men and women. Drug Alcohol Depend. 1991;28:121-8. This study shows that differential effects of marijuana on men and women are unclear, but male users have greater overall intracranial volume than females.

13. Block RI et al. Effects of frequent marijuana use on brain tissue volume and composition. Neuroreport. 2000;11:491-6.

14. Buckner JD, Zvolensky MJ, Schmidt NB. Cannabis-related impairment and social anxiety: the roles of gender and cannabis use motives. Addict Behav. 2012;37:1294-7.

15. Buckner JD, Mallott MA, Schmidt NB, Taylor J. Peer influence and gender differences in problematic cannabis use among individuals with social anxiety. Anxiety Disord. 2006;20:1087-102.

16. Cooper ZD, Haney M. Investigation of sex-dependent effects of cannabis in daily cannabis smokers. Drug Alcohol Depend. 2014;136:85-91.

17. Copersino ML, Boyd SJ, Tashkin DP, Huestis MA, Heishman SJ, Dermand JC, et al. Sociodemographic characteristics of cannabis smokers and the experience of cannabis withdrawal. Am J Drug Alcohol Abuse. 2010;36(6):311-9.

18. Cousijn J et al. Grey matter alterations associated with cannabis use: results of a VBM study in heavy cannabis users and healthy controls. NeuroImage. 2012;59:3845-51.

19.• Crane NA et al. Effects of cannabis on neurocognitive functioning: recent advances, neurodevelopmental influences, and sex differences. Neuropsychol Rev. 2013;23:117-37. This article provides a comprehensive review of sex effects of marijuana, largely in molecular and animal models, that supports the understanding of how marijuana affects neural structure and function.

20. Felton JW et al. Sex differences in self-report and behavioral measures of disinhibition predicting marijuana use across adolescence. Exp Clin Psychopharmacol. 2015;23:265-74.

21. Gillespie NA, Kendler KS, Neale MC. Psychometric modeling of cannabis initiation and use and the symptoms of cannabis abuse, dependence and withdrawal in a sample of male and female twins. Drug Alcohol Depend. 2011;118(2-3):166-72.

22. Guxens M, Nebot M, Ariza C. Age and sex differences in factors associated with the onset of cannabis use: a cohort study. Drug Alcohol Depend. 2007;88:234-43.

23. Johnson RM, Fairmana B, Gilreath T, Xuanc Z, Rothmanc EF, Parnhama T, et al. Past 15-year trends in adolescent marijuana use: differences by race/ethnicity and sex. Drug Alcohol Depend. $2015 ; 155: 8-15$. 
24. Jones AW, Holmgren A, Kugelberg FC. Driving under the influence of cannabis: a 10-year study of age and gender differences in the concentrations of tetrahydrocannabinol in blood. Addiction. 2008;103:452-61.

25. Lisdahl KM, Price JS. Increased marijuana use and gender predict poorer cognitive functioning in adolescents and emerging adults. $\mathrm{J}$ Int Neuropsychol Soc. 2012;18:678-88.

26. McDonald J et al. Effects of THC on behavioral measures of impulsivity in humans. Neuropsychopharmacology. 2003;28:1356-65.

27. McQueeny $\mathrm{T}$ et al. Gender effects on amygdala morphometry in adolescent marijuana users. Behav Brain Res. 2011;224:128-34.

28. Medina KL et al. Prefrontal cortex morphometry in abstinent adolescent marijuana users: subtle gender effects. Addict Biol. 2009; $14: 457-68$.

29. Noacka R, Höfler M, Lueken U. Cannabis use patterns and their association with DSM-IV cannabis dependence and gender. Eur Addict Res. 2011;17:321-8.

30. Pedersen W, Mastekaasa A, Wichstrøm L. Conduct problems and early cannabis initiation: a longitudinal study of gender differences. Addiction. 2001;96:415-31.

31. Pope HG et al. Evidence for a sex-specific residual effect of cannabis on visuospatial memory. Psychother Psychosom. 1997;66:17984.

32. Price JS et al. Effects of marijuana use on prefrontal and parietal volumes and cognition in emerging adults. Psychopharmacol (Berl). 2015;232:2939-50.

33. Roser $\mathrm{P}$ et al. Psychomotor performance in relation to acute oral administration of Delta(9)-tetrahydrocannabinol and standardized cannabis extract in healthy human subjects. Eur Arch Psychiatry Clin Neurosci. 2009;259:284-92.

34. Schepis TS, Desai RA, Cavallo DA, Smith AE, McFetridge A, Liss TB, et al. Gender differences in adolescent marijuana use and associated psychosocial characteristics. J Addict Med. 2011;5(1):65-73.

35. Skosnik PD et al. The effect of cannabis use and gender on the visual steady state evoked potential. Clin Neurophysiol. 2006; 117:144-56.

36. Tu AW, Ratner PA, Johnson JL. Gender differences in the correlates of Adolescents' cannabis use. Subst Use \& Misuse. 2008;43(10): 1438-63.

37.• Wetherill RR et al. Sex differences in associations between cannabis craving and neural responses to cannabis cues: implications for treatment. Exp Clin Psychopharmacol. 2015;23:238-46. This study shows that the neural craving response implicates different brain regions in men vs. women, with implications for differential treatment between the sexes.

38. Zalesky A et al. Effect of long-term cannabis use on axonal fibre connectivity. Brain. 2012;135:2245-55.

39. Brown TT, Dobs AS. Endocrine effects of marijuana. J Clin Pharmacol. 2002;42:90S-6.
40. Mendelson JH, Mello NK. Effects of marijuana on neuroendocrine hormones in human males and females. NIDA Res Monogr. 1984;44:97-114.

41. Mendelson $\mathrm{JH}$ et al. Acute effects of marijuana on pituitary and gonadal hormones during the periovulatory phase of the menstrual cycle. NIDA Res Monogr. 1984;55:24-31.

42. Kolodny RC et al. Depression of plasma testosterone levels after chronic intensive marihuana use. N Engl J Med. 1974;290:872-4.

43. Lenroot RK et al. Sexual dimorphism of brain developmental trajectories during childhood and adolescence. Neuroimage. 2007;36: 1065-73.

44. Giedd JN et al. Brain development during childhood and adolescence: a longitudinal MRI study. Nat Neurosci. 1999;2:861-3.

45. Schweinsburg AD et al. The influence of marijuana use on neurocognitive functioning in adolescents. Curr Drug Abuse Rev. 2008;1:99-111.

46. Wilson $\mathrm{W}$ et al. Brain morphological changes and early marijuana use. J Addict Dis. 2000;19:1-22.

47. Solinas $\mathrm{M}$ et al. Endocannabinoid system involvement in brain reward processes related to drug abuse. Pharmacol Res. 2007;56: 393-405.

48. Gonzalez R et al. Performance of young adult cannabis users on neurocognitive measures of impulsive behavior and their relationship to symptoms of cannabis use disorders. J Clin Exp Neuropsychol. 2012;34:962-76.

49. Cross CP et al. Sex differences in impulsivity: a meta-analysis. Psychol Bull. 2011;137:97-130.

50. Whelan $\mathrm{R}$ et al. Adolescent impulsivity phenotypes characterized by distinct brain networks. Nat Neurosci. 2012;15:920-5.

51. Dalley JW et al. Impulsivity, compulsivity, and top-down cognitive control. Neuron. 2011;69:680-94.

52. Filbey FM et al. Combined effects of marijuana and nicotine on memory performance and hippocampal volume. Behav Brain Res. 2015;293:46-53.

53. Chang L. Marijuana use is associated with a reorganized visualattention network and cerebellar hypoactivation. Brain. 2006;129: 1096-112.

54. Wada $Y$ et al. Gender differences in quantitative EEG at rest and during photic stimulation in normal young adults. Clin EEG. 1994;25:81-5.

55. Herlitz A et al. Gender differences in episodic memory. Mem Cognit. 1997;25:801-11.

56. Weiss EM et al. Sex differences in cognitive functions. Personal Individ Differ. 2003;35:863-75.

57. Filbey FM et al. Preliminary findings demonstrating latent effects of early adolescent marijuana use onset on cortical architecture. Dev Cognit Neurosci. 2015;16:16-22.

58. D'Souza DC et al. The psychotomimetic effects of intravenous delta-9-tetrahydrocannabinol in healthy individuals: implications for psychosis. Neuropsychopharmacology. 2004;29:1558-72. 